\title{
Chronic Allograft Dysfunction-Is There a Treatment?
}

\author{
A.C. Ferreira, H. Viana, F. Carvalho, J.R. Pinto, M.J. Galvão, F. Nolasco, and J.R. Santos
}

\begin{abstract}
Background. The major causes of renal transplant loss are death and chronic allograft dysfunction (CAD). The aims of this study were to determine the incidence of CAD in our population and the relation between allograft survival and immunosuppressive regimens.

Methods. We studied retrospectively 473 patients who received deceased donor kidney transplants with at least 1 allograft biopsy between January 1990 and May 2007. Clinical data included age, gender, biopsy data, and immunosuppression before and after kidney biopsy. Mean age was $45.4 \pm 12.7$ years including $65 \%$ males with a mean follow-up of $6.7 \pm 4.5$ years. CAD was observed in 177 of 473 biopsies: 48 patients showed interstitial fibrosis (IF); 101 chronic rejection (CR); 16 transplant glomerulopathy (TG); and 12, CR and TG. Mean follow-up since the discovery of the histologic feature was $60.5 \pm 50.5$
\end{abstract} months for IF; $38.3 \pm 40.8$ for CR, and $18.2 \pm 19.2$ for TG.

Results. CAD, which was more common in younger patients $(P=.03)$, correlated upon univariate and multivariate analysis with CKD stage 5d development $(P<.001)$. Deposition of $\mathrm{C} 4 \mathrm{~d}$ in peritubular capillaries was more frequent among CAD patients $(P=$ $.004)$, an association with particular relevance to recipients with $\mathrm{CR}(P=.02)$ and TG $(P<$ $.001)$. When we analyzed CAD subpopulation, we observed a positive correlation between allograft survival and immunosuppression modification after biopsy. Substitution of sirolimus (40/177) was shown in univariate, multivariate and Cox regression analyses to be a renal protector $(P<.002)$. Allograft survival was also correlated with initial mycophenolate mofetil versus azathioprine, $(62 / 177)$ immunosuppression $(P<.001)$.

Conclusion. CAD, a frequent histologic feature, may benefit from sirolimus conversion.

$\mathrm{R}$ ENAL TRANSPLANTATION has become the treatment of choice for CKD stage $5 \mathrm{~d}$. The major causes of renal transplant loss are death and chronic allograft dysfunction (CAD). ${ }^{1-3} \mathrm{CAD}$ is a generic term for renal allograft dysfunction in the absence of active acute rejection, drug toxicity, or other diseases. It is characterised by pathologic changes that involve all parts of renal parenchyma including the presence of interstitial fibrosis (IF) or tubular atrophy (TA). ${ }^{2}$ The Banff 2005 group included in this category the terms chronic rejection (CR) and transplant glomerulopathy (TG). ${ }^{1}$ Currently, there is no effective treatment for CAD, beside control of blood pressure. Various immunosuppressive regimens have been evaluated to limit the risk of CAD development or to limit its effects. $^{2,3}$ The aims of this study were to determine the incidence of CAD in our population and its relationship to allograft survival and immunosuppressive regimens.

\section{PATIENTS AND METHODS}

This retrospective study included 473 patients who received deceased donor kidney transplantation with at least 1 allograft biopsy between January 1990 and May 2007. All biopsies were performed based on clinical features; there were no protocol biopsies. Clinical data included age, gender, biopsy data, and immunosuppression before and after the kidney biopsy. According to histologic features, the patients were divided into 2 groups: the CAD group and a control group.

Univariate analysis (Spearman correlation) and multivariate analysis (linear regression, 95\% confidence interval [CI], using a

From the Nephrology Department, Hospital de Curry Cabral, Lisbon, Portugal.

Address reprint requests to Ana Carina da Costa Ferreira, Rua da Beneficência $n^{\circ} 8,1069$ Lisbon, Portugal. E-mail: karinadacostafer@hotmail.com

(C) 2009 by Elsevier Inc. All rights reserved. 360 Park Avenue South, New York, NY 10010-1710 
forward method) were performed with the SPSS system 15.0 (SPSS Inc., Chicago, IL); a $P<.05$ was considered significant.

\section{RESULTS}

Data for the variables recorded during the study are summarized in Table 1. CAD was present in 177 of 473 recipients $(37.4 \%)$. We subdivided these patients into 3 categories: patients with nonspecific IF/TA; patients with $\mathrm{CR}$, which was defined by IF/TA and pathologic changes in blood vessels; and patients with TG, defined by duplication of glomerular basement membrane. Using these histologic criteria, 48 patients had IF/AT; 101 CR; 16 TG; and 12 both $\mathrm{CR}$ and TG. Mean follow-up since histologic feature was $60.5 \pm 50.5$ months for IF; $38.3 \pm 40.8$ for CR; and $18.2 \pm$ 19.2 for TG. Since January 2005, PTC C4d deposition was performed on all kidney biopsies. Overall, 17 patients showed C4d deposition in PTC: 12 with CAD and 5 without.

Upon univariate analysis, CAD was more frequent among younger patients $(r=-0.98 ; P=.03)$ and correlated with PTC C4d deposition $(r=0.1 ; P=.004)$ and with allograft loss $(r=.2 ; P<.0001)$, particularly with CKD phase $5 \mathrm{~d}$ development $(r=0.27 ; P<.001)$. Upon multivariate analysis, there was a positive correlation between CAD and PTC C4d deposition $(P=.008 ; 95 \% \mathrm{CI}, 0.08-0.53)$ and CKD phase $5 \mathrm{~d}$ development $(P<.001 ; 95 \%$ CI, 0.2-0.4). Deposition of C4d in PTC was more frequent among CAD patients, an association with particular relevance to recipients with CR $(r=0.1 ; P=.02)$ and TG $(r=0.2 ; P<.001)$. Using linear regression, a correlation was only achieved in TG patients $(P<.001 ; 95 \% \mathrm{CI}, 0.1-0.3)$.

When we analyzed the CAD subpopulation, and at the end of follow-up, $47.5 \%(n=84)$ had developed CKD stage $5 \mathrm{~d}$ : $37 \%$ of IF/TA patients, $53.1 \%$ of CR patients, and $39.3 \%$ of TG patients. We observed a positive correlation between allograft loss and PTC C4d deposition upon both univariate and multivariate analyses $(r=0.4 ; P=.008 ; 95 \%$ CI, 0.2-0.9). Allograft survival positively correlated with immunosuppressive therapy modification after kidney biopsy, which occurred in 50 of 177 patients $(r=0.2 ; P=$ $.002)$. It was positively correlated with initial therapy using mycophenolate mofetil (MMF) versus azathioprine on both univariate and multivariate analyses $(62 / 177 ; r=0.3 ; P<$ $.001 ; 95 \%$ CI, 0.1-0.4). Introduction of sirolimus (40/177) in univariate, multivariate, and Cox regression analyses correlated with allograft survival $(r=0.3 ; P<.001 ; 95 \% \mathrm{CI},-0.1$ to $-0.5 ; P<.002$, respectively), namely a $20 \%$ better graft survival at 2 years after the index biopsy. The graft survival was $80 \%$ among those who received sirolimus versus $60 \%$ among those never treated with this drug $(P=.00029$, log-rank test).

\section{DISCUSSION}

The histologic diagnosis of CAD represents the final pathway of renal allograft damage. ${ }^{3}$ In this population, it represented a common histologic feature associated with loss of allograft function. Deposition of C4d in PTC was a risk factor for this outcome, as it was already described in other reports. ${ }^{4,5}$ During humoral rejection, antibody deposition in PTC resulted in activation of the classical pathway of complement generating $\mathrm{C} 4 \mathrm{~d}$, which binds covalently to PTC. This observation suggests that antibody-mediated rejection is a contributor to some forms of the chronic renal allograft lesion, formerly known as TG, which is a pathologic condition associated with poor allograft survival. ${ }^{6}$ Our study demonstrated that $\mathrm{C} 4 \mathrm{~d}$ deposition was frequent among the TG subgroup.

To reduce the incidence of CAD and allograft loss, it is necessary to find preventive and therapeutic measures. Therapeutic strategies have centred on avoidance of calcineurin inhibitors, but this strategy is associated with higher rates of acute rejection episodes, which are a known risk factor for development of CAD. ${ }^{3} \mathrm{MMF}$ has been used to reduce incidence of CAD or its effects. Both de novo use and conversion therapy are superior to the use of azathioprine. ${ }^{3,7}$ Sirolimus, an antiproliferative, non-nephrotoxic drug has been shown to be effective in several studies. ${ }^{2,3,8,9}$ In our study we did not observe any difference whether the initial immunosuppressive agent was cyclosplorine or tacrolimus maybe because both are nephrotoxins; both agents lead to vasoconstriction, which is reversible, and both produce transforming growth factor- $\beta$, leading to interstitial fibrosis, which is progressive and irreversible. Use of

Table 1. Clinical Data

\begin{tabular}{|c|c|c|c|c|}
\hline Variable & Patients $(n=473)$ & CAD Group $(n=177)$ & Control Group $(n=296)$ & $P$ \\
\hline Age (yrs) & $45.4 \pm 12.7$ & $43.5 \pm 13.2$ & $46.5 \pm 12.3$ & .03 \\
\hline \multicolumn{5}{|l|}{ Gender } \\
\hline Male & $69 \%$ & $61 \%$ & $67.6 \%$ & NS \\
\hline Female & $31 \%$ & $39 \%$ & $32.4 \%$ & NS \\
\hline Mean follow-up (yrs) & $6.7 \pm 4.5$ & $6.6 \pm 3.9$ & $6.8 \pm 4.9$ & NS \\
\hline Induction therapeutic (\%) & $15.4 \%(n=73)$ & $15.8 \%(n=28)$ & $15.2 \%(n=45)$ & NS \\
\hline Acute rejection episodes (\%) & $48.2 \%(n=228)$ & $51.4 \%(n=92)$ & $45.6 \%(n=136)$ & NS \\
\hline Cellular vs vascular & $191 ; 81$ & $79 ; 31$ & $112 ; 50$ & \\
\hline Allograft loss (\%) & $44.2 \%(n=209)$ & $55.9 \%(n=99)$ & $37.2 \%(n=110)$ & $<.001$ \\
\hline CKD stage $5 d(\%)$ & $31.1 \%(n=147)$ & $47.5 \%(n=84)$ & $21.3 \%(n=63)$ & $<.001$ \\
\hline Death $(\%)$ & $13.1 \%(n=62)$ & $8.5 \%(n=15)$ & $15.9 \%(n=47)$ & NS \\
\hline
\end{tabular}

Abbreviation: NS, not significant. 
MMF seemed to allow better allograft survival, and sirolimus was found to be a renal protector. Introduction of sirolimus proved to be effective in this population. We believe that this is due to its antiproliferative properties, leading to a reduced proliferation of fibroblasts and vascular smooth muscle cells and because the introduction of this drug allowed us to reduce or even withdrawn calcineurin inhibitors, sparing their effects.

\section{REFERENCES}

1. Solez K, Colvin RB, Racusen LC, et al: Banff '05 meeting report: differential diagnosis of chronic allograft injury and elimination of chronic allograft nephropathy. Am J Transplant 7:518, 2007

2. Afzali B, Taylor AL, Goldsmith JA: What can we do about chronic allograft nephropathy: role of immunosuppressive modulations. Kidney Int 1:145, 2005

3. Chapman JR, O'Connel PJ, Nankivell BJ: Chronic renal allograft dysfunction. J Am Soc Nephrol 16:3015, 2005
4. Haas M, Rahman MH, Racusen LC, et al: C4d and C3d staining in biopsies of ABO and HLA incompatible allografts: correlation with histologic findings. Am J Transplant 6:1829, 2006

5. Neto ED, Prado E, Beutel A, et al: C4d positive chronic rejection: a frequent entity with a poor outcome. Transplantation $84: 1391,2007$

6. Cosio FG, Gloor JM, Sethi S, et al: Transplant glomerulopathy. Am J Transplant 8:492, 2008

7. Merville P, Berge F, Deminiere C, et al: Lower incidence of chronic allograft nephropathy at 1 year post-transplantation in patients treated with mycophenolate mofetil. Am J Transplant 4:1769, 2004

8. Johnson RW, Kreis H, Oberbauer R, et al: Sirolimus allows early cyclosporine withdrawl in renal transplantation resulting in improved renal function and lower blood pressure. Transplantation 72:777, 2001

9. Kruger B, Fischereder M, Jauch KW, et al: Five year follow up after late conversion from calcineurin inhibitors to sirolimus in patients with chronic renal allograft dysfunction. Transplant Proc 39:518, 2007 